\title{
Assessment and contributions of grappling and punching forces in combat sports athletes: implications for the development of strength and conditioning
}

Luís MONTEIRO*

Universidade Lusófona de Humanidades e Tecnologias, Lisboa (Portugal)

\section{Abstract}

Training programs designed to improve strength in grappling and punching combat sports' athletes have become particularly relevant. The strength training plays a special role, with potential effect on high level performance, muscular balance and prevention of potential injuries. At this conference the protocols that can be used, depending on the level of the athletes and kind of combat sports they practica, will be detailed. Such information is vital for enhancing the scientific understanding of grappling, punching and consequently for the development of optimum strength and physical conditioning strategies.

Therefore, professionals must be acquainted with the latest information and be able to deal with the adequate criteria to prescribe safe and effective force training programs to combat sports athletes. This workshop will present the necessary criteria and practical tools in order to achieve the purposed goal:

(1) Initial considerations for the practice of strength training in Combat Sports: Explosive strength, power, plyometric, circuit training and physical conditioning;

(2) Strength training types depending on the intensity of the realization;

(3) Programming strength training of the grappler and the puncher; and

(4) Design, implementation and evaluation of strength protocols.

\section{Introduction}

Performance during human movements is highly related to force and velocity muscle capacities. Those capacities are highly developed in elite athletes practicing power-oriented sports (Giroux, et al., 2015). However, it is still unclear whether in the different combat sports between their force and velocity generating capacities constitute an optimal profile. In this workshop we aim to determine the effect of elite sport background on the force-velocity relationship in each combat sport, and evaluate the level of optimization of these profiles.

Grapplers need high levels of dynamic [concentric (CON) and eccentric (ECC)] and isometric strength and endurance. Wrestlers and judo athletes have sufficient levels of dynamic muscular strength, for example, absolute strength is greater in heavier athletes, whereas relative strength is greater in smaller athletes. Wrestlers have high levels of strength in neck flexion, extension, lateral rotation, and sport-specific (bear hug) movements (Iwai, et al., 2008). In comparison, wrestlers have greater trunk flexion and extension strength (and larger rectus abdominis muscle size) but less oblique and quadratus lumborum muscle size than judo athletes of similar mass (Demirkan, et al., 2015). Studies have shown a mean 1 repetition maximum (1RM) bench press of 96-160 $\mathrm{kg}$ and 
squat of 104-185 kg in male judo athletes (Sbriccoli, et al., 2007). Successful wrestlers have greater isokinetic strength than less-successful wrestlers. Isometric strength is needed for grabbing and holding an opponent.

Competitive judo can be described as a combative high-intensity sport in which the athlete attempts to throw the opponent onto his/her back or to control him/her during groundwork combat (Franchini, et al., 2007).

Many actions during combat are highly explosive and require strength and coordination to overcome the adversary through rapid execution of technical maneuvers (Chaabène, et al., 2015). The judo movement structure is very demanding for most muscle groups, so contractile forces play an important role in executing judo throwing techniques (Monteiro, et al., 2011). Therefore, the extensor and flexor strength of thigh and shoulder muscles could discriminate between successful and less successful athletes in judo competition.

Judo has high physical demands and requires the athlete to be in an optimal physical fitness (Franchini, et al., 2007). Most matches last approximately 3-4 minutes, with 20 to 30-second intermittent bursts of high-intensity activity. Because of these judo combat characteristics, elite judokas have high-level muscular strength depending on weight class, and they score very well on pull-up, push-up, and sit-up endurance tests.

Wrestling, as a sport, is extremely dynamic by nature, being characterized by sudden explosive attacks and counterattacks that are executed repeatedly at a high intensity and alternated with submaximal work for duration of up to 6 minutes (Hubner-Wozniak, et al., 2004; Mirzaei, et al., 2009). Because of the nature of this activity, wrestling demands several specific characteristics, including maximal strength, aerobic endurance, and anaerobic capabilities to achieve success in competition (Zi-Hong, et al., 2013). The current scoring system promotes a quick explosive action style as well (Hubner-Wozniak, et al., 2004). The anaerobic system provides the short quick bursts of maximal power during the match, whereas the aerobic system contributes to the wrestler's ability to sustain effort for the duration of the match (Callan, et al., 2000; Karnincic, et al., 2009).

During karate kumite competition, the two opponents execute punching and kicking karate skills. Punching techniques are applied more frequently than kicking techniques. Thus, to be successful, the athlete must present a rapid programming of adequate offensive and defensive techniques in response to the opponent actions. Still, due to the importance of speed-power related abilities in karate performance, strength and conditioning professionals are encouraged to develop neuromuscular programs capable of improving lower and upper limbs muscle power in top-level karate athletes. Finally, coaches and sports scientists should also determine the specific demand of their athletes' matches to improve training organization and to identify aspects needing improvement (Chaabène, et al., 2015).

In boxing, athletes need to punch at different distances from the target in both training and competition; thus, investigating the effect of distance variation (self-selected vs. predetermined)on impact is important to establish the best training strategy for enhancing boxers' technical and tactical skills. Additionally, identification of the strength-power qualities more associated with punching impact in the most executed strokes (i.e., jabs and crosses) is essential for developing better neuromuscular training methods and, as a consequence, for improving the boxers' competitiveness.

Based on a previous research (Lenetsky, et al., 2013) that reported high correlations between punching acceleration and selected strength-power variables, and considering the crucial role of the g-forces (i.e., acceleration due to gravity) on the impact measurements, we hypothesized that the stronger/more powerful athletes would perform better in both punching techniques.

\section{Discussion}

Because of the large to very large correlations found between strength-power measurements in the lower and upper extremities and the impact forces produced/applied by elite and amateur combat sports when executing the skills throws, punches or grapples, strength and conditioning coaches 
are strongly encouraged to implement specific training strategies to improve performance in such variables. Both the upper and lower limbs have to be effective in applying high levels of force at high velocities for generating superior levels of muscle power (Kawamori \& Haff, 2004). Accordingly, basic power exercises, such as the JS, BP, and BT, using a range of loads capable of increasing the power outputs, could be implemented to enhance the impact of the techniques. In addition, athletes should develop maximal strength by using methods able to elicit positive adaptations in the lower limbs, focusing on methods that induce neural adaptations rather than hypertrophic responses. Further studies should investigate the possible chronic and specific adaptations of using neuromuscular stimulus (i.e., strength power exercises, plyometrics, etc.) as the priming for the execution of punches, because recent research has shown the effectiveness of complex or contrast methods in other sports specialties, based on the postactivation potentiation phenomenon (Ratamess, 2011).

Vertical jumps (i.e., SJ and CMJ) are commonly used by strength and conditioning coaches to assess lower limb explosiveness in athletes from different sports disciplines (Ratamess, 2011). To some extent, this can be explained by the extensive list of correlations presented between these exercises and actual athletes' performance (Monteiro, et al., 2011). In combat sports, vertical jumping ability has been shown to be significantly related to specific fighting techniques (i.e., punching acceleration in karate, throwing techniques in judo) (Loturco, et al., 2016).

Previous findings suggest that both SJ and CMJ exert important influence on punching impact (Ratamess, 2011; Loturco, 2016), being able to explain 75\% of the magnitudes of forces applied by elite boxers during jab and cross executions. It should be noted that the strength power qualities play an important role in vertical jumping performance and, even in this group of combat athletes, the subjects able to perform better in squats and JS are also able to jump higher (Lenetsky, et al., 2013). Taken together, these facts strongly suggest the inclusion of plyometric exercises in training routines that aim to increase fighting-specific neuromechanical capacities in elite "strikers" (i.e., boxers, kickboxers, judokas, Muay-Thai athletes, karate athletes, taekwondo, etc.) because of their effectiveness in eliciting positive adaptations in a wide range of factors related to power performance (e.g., stretch shortening cycle and muscle recruitment pattern) (Lenetsky, et al., 2013).

Performance diagnosis is the process of determining an athlete's level of development of distinct strength, power, speed, endurance and other qualities. The evaluation is an important aspect of the training program, and a discussion of the process of determining, assessing and monitoring performance qualities critical to the target combat sports. The assessment and interpretation through the performance diagnosis is the neuromuscular key component that characterize performance as well as the discriminating and evaluating components, invaluable for athlete monitoring/program, for combative sports and study the impact of repeated physical contact.

We will be presented with the latest Measurement Technology (Isocontrol, Chronojump and MG), where will be showed the practice evaluation and discussion of: (1) Displacement (distance) measurement; (2) Force measurement; (3) Balance; (4) Symmetry; Contractile proprieties muscles. We also will analyze the Counter movement jump (Chronojump): (1) Displacement; (2) Calculate jump height; (2) peak velocity; and (3) power output.

\section{Conclusion}

In conclusion, this workshop tries to characterize the profiles of each combat sport together with their optimization level on such a wide cohort of elite athletes. Trained for power oriented sports, these athletes displayed differently profiles, reflecting the specific adaptations inherent to the chronic practice of their activity. These findings confirm the influence of training background and sport activity on the force and velocity capacities balance. Finally, the present workshop gathers some evidence that elite athletes do not systematically present optimal F-V profiles, suggesting some interesting perspectives to improve athletic performance.

Finally, coaches and sports scientists should also determine the specific demand of their athletes' matches to improve training organization and to identify aspects needing improvement. 
In addition, to apply the different and new training programs in the future we must discuss some aspects:

(1) Online data collection - wireless;

(2) Real-time reporting;

(3) Web-based report and support;

(4) Field monitoring - training and testing; and

(5) Education - on-line, extensive, pervasive, accurate, based in science, relevant.

\section{References}

Callan, S.D., Brunner, D.M., Devolve, K.L., Mulligan, S.E., Hesson, J., Wilber, R.L., \& Kearney, J.T. (2000). Physiological profiles of elite freestyle wrestlers. Journal of Strength and Conditioning Research, 14(2), 162-169.

Chaabène, H., Hachana, Y., Franchini, E., Mkaoue, B., \& Chamari, K. (2012). Physical and Physiological Profile of Elite Karate Athletes. Sports Medicine, 42(10), 829-843.

Demirkan, E., Koz, M., Kutlu, M., \& Favre, M. (2015). Comparison of physical and physiological profiles in elite and amateur young wrestlers. Journal of Strength and Conditioning Research, 29(7), 1876-1883.

Franchini, E., Nunes, A.V., Moraes, J.M., \& Del Vecchio, F.B. (2007). Physical fitness and anthropometrical profile of the Brazilian male judo team. Journal of physiological anthropology, 26(2), 59-67.

Giroux, C., Rabita, G., Chollet, D., \& Guilhem, G. (2015). Optimal Balance Between Force and Velocity Differs Among World-Class Athletes. Journal of Applied Biomechanics, 32(1), 59-68

Hubner-Wozniak, E., Kosmol, A., Lutoslawska, G., \& Bem, E.Z. (2004). Anaerobic performance of arms and legs in male and female free style wrestlers. Journal of science and medicine in sport, $7(4), 473-480$.

Iwai. K., Okada, T., Nakazato, K., Fujimoto, H., Yamamoto, Y., \& Nakajima, H. (2008). Sport specific characteristics of trunk muscles in collegiate wrestlers and judokas. Journal of Strength and Conditioning Research, 22(2), 350-358.

Karnincic, H., Tocilj, Z., Uljevic, O., \& Erceg, M. (2009). Lactate profile during Greco-Roman wrestling matchx. Journal of sports science and medicine, 8(CSSI3), 17-19.

Kawamori, N., \& Haff, G.G. (2004). The optimal training load for the development of muscular power. Journal of Strength and Conditioning Research, 18(3), 675-84.

Lenetsky, S., Harris, N., \& Brughelli, M. (2013). Assessment and Contributors of Punching Forces in Combat Sports Athletes: Implications for Strength and Conditioning. Strength and Conditioning Journal, 35(2), 1-7.

Loturco, I., Nakamura, F.Y., Artioli, G.G., Kobal, R., Kitamura, K., Cal Abad, C.C., Cruz, I.F., Romano, F., Pereira, L.A., \& Franchini, E. (2016). Strength and power qualities are highly associated with punching impact in elite amateur boxers. Journal of Strength and Conditioning Research, 30(1): 109-116.

Mirzaei, B., Curby, D.G., Rahmani-Nia, F., \& Moghadasi, M. (2009). Physiological profile of elite Iranian junior freestyle wrestlers. Journal of Strength and Conditioning Research, 23(8), 23392344.

Monteiro, L., Massuça, L., García-García, J., Carratalá, V., \& Proença, J. (2011). Plyometric muscular action tests in judo - and non-judo athletes. Isokinetics and Exercise Science, 19(4), 287-293.

Ratamess, N. (2011). Strength and Conditioning for Grappling Sports. Strength Conditioning Journal. 33(6), 18-24.

Sbriccoli, P., Bazzucchi, I., Di Mario, A., Marzattinocci, G., \& Felici, F. (2007). Assessment of maximal cardiorespiratory performance and muscle power in the Italian Olympic judoka. Journal of Strength and Conditioning Research, 21(3), 738-744.

Tabben, M., Chaouachi, A., Mahfoudhi, M., Aloui, A., Habacha, H., Tourny, C., \& Franchini, E. (2014). Physical and physiological characteristics of high-level combat sport athletes. Journal of Combat Sports and Martial Arts, 1(2), 5, 1-5. 
Zi-Hong, H., Lian-Shi, F., Hao-Jie, Z., Kui-Yuan, X., Feng-Tang, C., Da-Lang, T., Ming-Yi, L., Lucia, A., \& Fleck, SJ. (2013). Physiological profile of elite Chinese female wrestlers. Journal of Strength and Conditioning Research, 27(9), 2374-2395.

Key words: Combat sports; grappling; punching; strength and conditioning; training control and evaluation. 